\title{
INVESTIGACIÓN
}

Recibido: 29/06/2016 --- Aceptado: 24/09/2016 --- Publicado: 15/03/2017

\section{USO DE GÉNEROS PERIODÍSTICOS, VALORES NOTICIA Y FUENTES DE INFORMACIÓN EN LOS MEDIOS DE COMUNICACIÓN DE SANTIAGO DE CUBA}

Viviana Muñiz Zúñiga1: Universidad de Oriente, Cuba.

vivita@uo.edu.cu

Rafael Ángel Fonseca Valido: Universidad de Oriente, Cuba.

fonseca@uo.edu.cu

\section{RESUMEN}

La presente constituye un estudio mixto, que caracteriza el uso de los géneros periodísticos, los valores noticia y las fuentes de información en los medios provinciales de Santiago de Cuba. Para ello se emplearon métodos como el de Análisis-síntesis y el Inductivo-deductivo; y se realizó un análisis de contenido utilizando la semana construida durante 9 semanas a los órganos de prensa provinciales. Esos datos se triangularon con entrevistas y encuestas a los periodistas que en ellos laboran, para determinar qué opinión tienen sobre estos elementos. Con el análisis de los resultados se pudo establecer que se emplean la información, el reportaje y el comentario como géneros, lo que coincide con la presencia de valores noticia como la actualidad, el interés humano y la proximidad. Las rutinas de uso de estos elementos están vinculadas con el perfil del medio analizado, y sus procedimientos para elaborar los trabajos periodísticos. En la provincia los periodistas emplean más las fuentes documentales e institucionales, lo que se debe a la credibilidad de estas para lograr un producto de mayor calidad.

\section{PALABRAS CLAVE}

Géneros periodísticos - valores noticia - fuentes de información - construcción de la noticia - rutinas productivas - Cuba.

\section{USE OF JOURNALISTIC GENRES, VALUES NEWS AND INFORMATION SOURCES IN THE MEDIA OF SANTIAGO DE CUBA.}

\footnotetext{
1Viviana Muñiz Zúñiga: Licenciada en Periodismo. Investiga desde el 2013 los procesos de construcción y establecimiento de la agenda mediática en Cuba; la implementación de las Política Informativa y Editorial y la construcción de la noticia en los medios cubanos.

vivita@uo.edu.cu
} 


\begin{abstract}
This is a mixed study that characterizes the use of journalistic genres, values news and information sources in the provincial media Santiago de Cuba. For this purpose methods such as analysis-synthesis and inductive-deductive they were used; and a content analysis was performed using the constructed week for 9 weeks to press provincial bodies. These data were triangulated with interviews and surveys of journalists working in them to determine what their opinion of these elements. With the analysis of the results was established that information, reportage and commentary as genres are used, which coincides with the presence of news values as today, human interest and proximity. The routine use of these elements is linked to the profile of the average analyzed, and processes for making journalistic work. In the province employ more journalist documentary and institutional sources, which is due to the credibility of these to achieve a higher quality product.
\end{abstract}

\title{
KEY WORDS
}

Journalistic genres, news values, sources of information, construction news, productive routines, Cuba.

\section{USO DOS GÊNEROS JORNALÍSTICOS, VALORES NOTÍCIA E FONTES DE INFORMAÇÃO NOS MEIOS DE COMUNICAÇÃO DE SANTIAGO DE CUBA.}

\section{RESUMO}

O presente constitui um estudo misto, que caracteriza o uso dos gêneros jornalísticos, os valores notícia e as fontes de informação nos meios provinciais de Santiago de Cuba. Para isso usaram métodos como o de analises-síntese e o indutivo-dedutivo; e realizou se uma analise do conteúdo utilizando a semana construída durante nove semanas aos órgãos da imprensa provinciais. Esses dados se triangularam com entrevistas e encostas aos jornalistas que neles trabalhavam para determinar que opiniões tinham sobre esses elementos. Com a analises dos resultados puderam estabelecer que usam a informação, a reportagem e o comentário como gêneros, o que coincide com a presença de valores noticia como a atualidade, o interesse humano e a proximidade. As rotinas de uso desses elementos estão vinculadas com perfil do meio analisado, e seus procedimentos para elaborar os trabalhos jornalísticos. $\mathrm{Na}$ província os jornalistas usam mais as fontes documentais e institucionais, devido à credibilidade destas, para lograr um produto de maior qualidade.

\section{PALAVRAS CHAVE}

Gêneros jornalísticos - Valor notícia - Fontes de informação - Construção da notícia Rotinas produtivas - Cuba 


\section{Cómo citar el artículo}

Muñiz Zúñiga, V.; Fonseca Valido, R. A. (2017). Uso de géneros periodísticos, valores noticia y fuentes de información en los medios de comunicación de Santiago de Cuba. [Use of journalistic genres, news values and information sources in the media in Santiago de Cuba]. Vivat Academia. Revista de Comunicación, $n^{\circ} 138,120-140$. doi http://dx.doi.org/10.15178/va.2017.138.120-140. Recuperado de http:/ / vivatacademia.net/index.php/vivat/article/view/1018

\section{INTRODUCCIÓN}

La calidad periodística en diferentes medios de comunicación es objeto de estudio en no pocas investigaciones desarrolladas durante los últimos 5 años (Mompart y Sampio, 2013; Mompart, Lozano y Sampio, 2013; De la Piscina, Gorosarri, Zabalondo y Agirre, 2014; Aguirre y Bernal, 2015; De la Torre y Téramo, 2015; Mompart, Lozano y Sampio, 2015; Reyes, González, Torrijos y Gordillo, 2015; Carballosa, Pavón y Rodríguez, 2016). Estos estudios han evaluado el producto mediático a partir de diferentes indicadores como el uso de los valores noticia y los criterios de noticiabilidad, las fuentes de información y los géneros periodísticos; las rutinas productivas y la concepción de los profesionales del gremio acerca del trabajo que realizan.

Mompart y Sampio (2013) explican que la necesidad de abordar la calidad periodística ha estado vinculada con tradiciones metodológicas diversas, que en Norteamérica tienen una naturaleza comercial, a diferencia de los estudios europeos que son más informativos, con una idea ligada a la cantidad de información y el espacio dedicado por un medio a un conjunto de hechos. Por ejemplo, en España se edita el Informe Anual de la Profesión Periodística (Asociación de la prensa de Madrid, 2013) en el que se refleja el comportamiento de factores como el mercado laboral, los escenarios de actuación profesional de los periodistas a partir de la crisis económica, las condiciones laborales y la actitud hacia las transformaciones que las Tecnologías de la Información y las Comunicaciones han impuesto.

En Cuba existen estudios que han evaluado el uso de elementos que permiten enriquecer el producto periodístico (Rosquete, 2010; Colunga, 2011; Toledo, Medina y Hernández, 2015; Carballosa, Pavón y Rodríguez, 2016; Vicente, Rojas y Vicente, 2016) aunque urgen estudios longitudinales que apunten hacia una sistematización de dichos factores en órganos de prensa territoriales (provinciales).

Por esa razón, esta investigación profundiza en el empleo de los géneros periodísticos, los valores noticia y las fuentes de información en medios audiovisuales e impresos de la provincia Santiago de Cuba, utilizando para ello el método de semana construida, a través del cual se seleccionaron 9 semanas del año 2015. Ese resultado cuantitativo fue triangulado con entrevistas y una encuesta sobre 
la percepción que tienen los periodistas sobre el uso de estos elementos. En ese sentido, se plantea como problema de investigación ¿Qué características posee el uso de los géneros periodísticos, los valores noticia y las fuentes de información en los órganos de prensa provinciales de Santiago de Cuba durante el año 2015?

\section{OBJETIVOS}

El objetivo general del presente estudio es Caracterizar el uso de los géneros periodísticos, los valores noticia y las fuentes de información en los órganos de prensa provinciales de Santiago de Cuba durante el año 2015. Para dar cumplimiento a ese propósito macro se establecieron las siguientes tareas de investigación $u$ objetivos específicos:

1. Determinar el empleo de los géneros periodísticos informativos, de opinión e interpretativos en los trabajos publicados por los medios de comunicación analizados.

2. Determinar el uso de los valores noticia en la muestra analizada.

3. Determinar el empleo de fuentes de información institucionales, personales y documentales en los medios de comunicación escogidos.

4. Evaluar la percepción que tienen los periodistas respecto a las rutinas de uso de los géneros periodísticos, los valores noticia y las fuentes de información en sus respectivos medios.

\section{METODOLOGÍA}

El presente estudio utiliza un diseño mixto de investigación, pues en él confluyen procedimientos cuantitativos y cualitativos para la caracterización del uso de los géneros periodísticos, los valores noticia y las fuentes de información en los medios santiagueros. Por ello se aplican métodos como el de Análisis-síntesis, en el procesamiento de la bibliografía consultada; y el Inductivo-deductivo en el establecimiento de generalidades tomando en cuenta las particularidades y viceversa.

En función de ello, se emplearon técnicas como el análisis de contenido cuantitativo a los medios, sobre el cual se realizaron cálculos porcentuales y la prueba binomial no paramétrica que posibilitó establecer los niveles de significación de los valores encontrados. Los resultados de este análisis se triangularon con la aplicación de una encuesta a una muestra de 64 periodistas en activo de la provincia, en la cual se les preguntó sobre el empleo de los géneros periodísticos, los valores noticia y las fuentes de información en los trabajos que publicaban en sus respectivos medios. Posteriormente se realizó una entrevista semi-estandarizada a 38 periodistas para realizar una valoración cualitativa y se utilizó la observación participante por el período de dos meses en los medios de comunicación para evaluar algunas rutinas de uso de estos elementos.

Como apuntábamos previamente, para el análisis de contenido se empleó el método de semana construida, que "permite obtener una muestra aleatoria de la información de los medios ampliando el rango de su selección. Esto evita distorsiones propias de un momento 
informativo reducido, al mismo tiempo que suele conservar las marcas de la pauta editorial" (Teramo, 2006, p. 59). Con esta metodología se analizaron durante 9 semanas 349 trabajos en la emisora CMKC, tomados del noticiero diario Reportes. En el telecentro Tele Turquino se evaluaron 246 trabajos publicados en los programas informativos Tele Noticias, Revista Santiago y Última hora; y en el periódico Sierra Maestra se tomaron 198 trabajos de su versión impresa.

\section{RESULTADOS Y DISCUSIÓN}

\subsection{Apuntes teóricos}

La realidad ofrece a la mirada del periodista un amplio diapasón de contenidos, sobre los cuales discernir su importancia para las personas que escribe. Así lo explica el teórico Walter Lippmann (1969) quien señaló que la información es tan difícil de obtener que está más allá de los recursos de la prensa diaria. Por ello, algunos autores (Gomis, 1991; Pineda y Molero, 2015; Vicente, Rojas y Vicente, 2016) plantean que el rol de los medios de comunicación como mediadores sociales entre los hechos de la realidad y las personas es más completo cuanto mayor es su capacidad o iniciativa en la difusión de estos. En ese sentido, el autor (Gomis, 1990) refiere que un medio puede "generar" hechos cuando potencia una situación determinada en detrimento de otra, encuadrando y estableciendo una agenda temática (Mompart, Lozano y Sampio, 2013; De la Piscina, Gorosarri, Zabalondo y Agirre, 2014).

Grossi (1983) apuntó que los medios son co-productores del producto periodístico, porque no se limitan a transmitir linealmente los acontecimientos inabarcables (McCombs, 2006) para la sociedad; destacando el papel del gatekeeper (Alsina, 1993) en la selección y jerarquización del mensaje informativo. En esta dirección los géneros periodísticos, los valores noticia y las fuentes de información constituyen tres elementos importantes en el encuadre del hecho noticioso, y constituyen objeto de análisis de numerosas investigaciones que se realizan propuestas enfocadas hacia la calidad periodística y la evaluación del producto mediático (Aguirre y Bernal, 2015; Hernández, 2015; Mompart, Lozano y Sampio, 2015; Carballosa, Pavón y Rodríguez, 2016).

Los tres elementos integran el complejo proceso de producción noticiosa, que para los autores Golding y Elliot (1979) comienza con la planificación, la recopilación de los datos, la selección y jerarquización de los elementos y la producción del material.

\subsubsection{Los géneros periodísticos}

Algunos autores (Cardoso, 2008) reconocen como géneros periodísticos a determinadas formas básicas que caracterizan el contenido y su estructura, así como los métodos y medios lingüísticos, literarios y artísticos de elaboración del discurso en correspondencia con las funciones e intencionalidad asumidas por el órgano de prensa y el periodista. En ese sentido otros autores (Serrano, 1982) plantean que la principal diferenciación que se puede observar en estos géneros proviene del objetivo del producto comunicativo que se elabora, y por tanto, de la función social que este cumplirá: si es informativa, educativa, interpretativa, de opinión, lúdica o 
instrumental (Cardoso, 2008).

En ese sentido, la tendencia a la hibridación entre géneros periodísticos es una característica inherente a la evolución de la prensa (Cardoso, 2008; Ribeiro, 2016), que se acentúa en el caso de los medios digitales, gracias a la hipertextualidad, la multimedialidad y la interactividad:

"Por una parte, con la hipertextualidad, se intensifica la permeabilidad entre textos periodísticos y se extiende la capacidad de asociarse entre sí (...) Por otra parte, la emergencia de la multimedialidad trae consigo la combinación de lenguajes desarrollados de manera independiente durante décadas en los diferentes medios de comunicación" (Ribeiro, 2016, p. 267).

El estudio de Ribeiro (2016) analiza el uso de los géneros ciberperiodísticos en blogs de profesionales de la prensa, sistematizando la evolución de la nomenclatura de estos desde el año 2000. Una de las tendencias de investigación y realización de los géneros periodísticos es el llamado "Periodismo lento" (Cedillo y Carretero, 2015, p. 37), que "emerge como reacción a la tendencia dominante a la inmediatez y la primicia y que invita a repensar los tiempos necesarios para producir y consumir una información rigurosa, creativa y de calidad". Según los autores el futuro de este tipo de práctica estriba en una noticia breve, barata y fácil de producir con el apoyo de las tecnologías: que convivirá con el periodismo especializado que empleará textos más extensos con una elevada factura estética. A pesar de que esta tendencia podría resultar más costosa que la experiencia tradicional, lograría una mayor compenetración con las audiencias, al brindarles un producto con un mayor nivel de acabado en la redacción y confección.

\subsubsection{El uso de las fuentes de información}

Lorenzo Gomis (1991) explica que por lo general los hechos a los que se les brinda cobertura mediática han sido previamente escogidos por los interesados en que ese hecho se conozca, criterio que han corroborado estudios prácticos en diferentes medios de comunicación (Alcaraz, 2012; Ibáñez, Alonso y Hernández, 2015; Macías, 2015). En tal sentido, existen razones por las cuales la relación ocurre de este modo: la gratuidad - en algunos casos- en la difusión del hecho, el interés de la fuente por divulgar hechos que considera pueden convertirse en noticia, y por lo tanto, interesarle al público (Gomis, 1991).

Autores como León Sigal (1973) y Brown, Bybee, Wearden y Straughan (1987) han demostrado que las informaciones llegan por lo general a través de comunicados, notas de prensa, y otros canales habituales; por lo que confirmaron que los medios tienen menos control sobre la elaboración de sus agendas del que realmente piensan. En ese sentido, se destaca la capacidad de las fuentes políticas para establecer las prioridades temáticas, mediante la "producción artificial de acontecimientos" (Grossi, 1981), que el periodista utiliza e imbrica con la relevancia del tema a nivel público. Para este autor la relación fuente-periodista se manifiesta de forma ambivalente: las fuentes regulan el equilibrio y representan los confines mutables entre los medios y la realidad. En este punto es preciso señalar que esta relación posee un alto componente de consenso y negociación ideológica, pues la fuente 
querrá que el acontecimiento se refleje de una manera específica, y el periodista posee un doble compromiso social.

Algunos autores (Reyes, 2006) coinciden en que las fuentes pueden ser clasificadas como documentales y no documentales. Entre las primeras se registran importantes y valiosos documentos que forman parte de la cultura y la memoria histórica que regularmente contienen información textual. Debido a la inmediatez propia del oficio del periodista, la revisión bibliográfico documental que posibilita Internet constituye una herramienta frecuente en los trabajos diarios. Las fuentes no documentales, como su nombre indica, pueden encontrarse en cualquier otro formato que no sea un documento, ya sea una grabación, foto, etc. Las fuentes de información también pueden ser clasificadas como oficiales y extraoficiales (Alsina, 1993).

El establecimiento y validación de una estrategia de fuentes queda cristalizada en el uso cotidiano de las mismas. A diario, los periodistas acuden a sus fuentes, y en la mayoría de los casos, aquellas fuentes institucionalizadas $u$ oficiales que refuerzan y legitiman la veracidad del mensaje que se brinda (García, 2013). Otras clasificaciones se refieren al nivel de relevancia de la fuente o al interés de las mismas por informar algo (Cardoso, 2008). Las fuentes interesadas en informar buscan al medio, o alguien insertado dentro del mismo que le comunique con el periodista que atiende el tema relacionado a su información. Este tipo de fuente pueden ser personas comunes, especialistas en menor grado, artistas, centros de investigación, entre otros. Según Cardoso (2008) se puede acceder a las fuentes por tres vías diferentes:

- Búsqueda orientada por la institución

- Divulgadores o departamentos de prensa

- Contacto personal

Mantener con las fuentes una buena relación es crucial para el aviso oportuno y la entrega de información de primera mano y de máxima calidad. Pero esa relación "buena" debe mantener una distancia profesional entre ambos -periodistas y fuentespues si se pierde la distancia profesional con las fuentes, con las instituciones y con los funcionarios, se crean distorsiones que impiden saber dónde está tu identidad y tu misión. Eso rebaja la capacidad de autorregulación y se vuelve entonces al concepto del periodista como "vocero del organismo" (García, 2013). El uso constante de una misma fuente legitima esta ante la sociedad, le confiere cierto status y relevancia social.

\subsubsection{Los valores noticia}

La autora del texto Periodismo, noticia y noticiabilidad (Martini, 2000) define la noticia como la particular construcción del discurso de la información que narra eventos factuales generalmente públicos. Como imagen del presente social (Gomis, 1991) es el relato de un suceso que implica o afecta a individuos de la sociedad.

Si se toman en cuenta los valores que rigen la noticiabilidad, la noticia puede ser definida como la construcción periodística de un acontecimiento cuya novedad, imprevisibilidad y efectos futuros sobre la sociedad lo ubican públicamente para su reconocimiento (Martini, 2000). Los valores noticia han sido utilizados por los 
periodistas como uno de los filtros más importantes que debe superar un hecho para convertirse en noticia. "Los valores-noticia son (...) reglas prácticas que incluyen un corpus de conocimientos profesionales que implícitamente, y a menudo explícitamente, explican y dirigen los procesos de trabajo en la redacción" (Golding y Elliott, 1979, p. 59). Están sujetos al contexto comunicativo (Orosa y Santorum, 2012) y facilitan el rápido desarrollo de los procesos productivos al interior de las redacciones periodísticas. Es válido aclarar que un acontecimiento no necesariamente tiene que presentar todos estos valores, aunque mientras más valores incluyan, mayores serán las ventajas de incorporarse al proceso productivo de los medios y convertirse en noticia.

Algunos de los más abordados (Martini, 2000) son la actualidad, la inmediatez, la veracidad, el interés colectivo y humano, la prominencia, la originalidad, y la proximidad. Sin embargo, pueden señalarse otras cualidades que posee el acontecimiento en relación con los procesos productivos:

La comprensión e inteligibilidad de un acontecimiento. Un acontecimiento comprensible por lo general permite mostrar resultados: Gomis (1991) agrega que los resultados constituyen el rasgo ideal de una noticia, porque de esta manera, un hecho se define como tal, es público y tiene repercusiones en el futuro.

La credibilidad.

La brevedad, que se relaciona con las cualidades anotadas antes, y consiste en la posibilidad de construir una noticia en pocas líneas o al menos de manera directa.

La periodicidad. Los hechos que son habituales y tienen una aparición periódica en los medios son más fáciles de construir y también de interpretar por el público que los consume.

La exclusividad o la primicia permite el éxito que significa la captura de la novedad antes que otros lo hagan, sino que implica la capacidad para contactar fuentes legítimas y privilegiadas y para leer de manera productiva los hechos en la realidad (Martini, 2000).

\subsection{Uso de los géneros periodísticos y los valores noticia en los medios santiagueros}

En el caso de la emisora CMKC el género más utilizado fue la Información, con un $73,9 \%$ de los trabajos, seguida del Comentario $(11,2 \%)$, la entrevista $(8,0 \%)$ y el Reportaje $(4,3 \%)$. Este resultado indica que en la emisora se privilegió en gran medida la interpretación de los acontecimientos a través de los hechos, lo que está relacionado estrechamente con el formato del medio: la radio ofrece recursos sonoros y el factor tiempo es menos limitado que en la televisión o el espacio del periódico (solo 8 páginas en formato tabloide una vez a la semana). Esto se corresponde con el uso de valores noticia como la actualidad ( $39,0 \%$ de las menciones), la proximidad $(37,8 \%)$ y el interés humano (11,6\%). Como se muestra en la Figura 1 ambos elementos se manifiestan con relativa estabilidad temporal, pues apenas se producen cambios cuantitativos según reveló el análisis de contenido. 
Figura 1 Uso de los géneros periodísticos y valores noticia en la emisora CMKC según las semanas de estudio

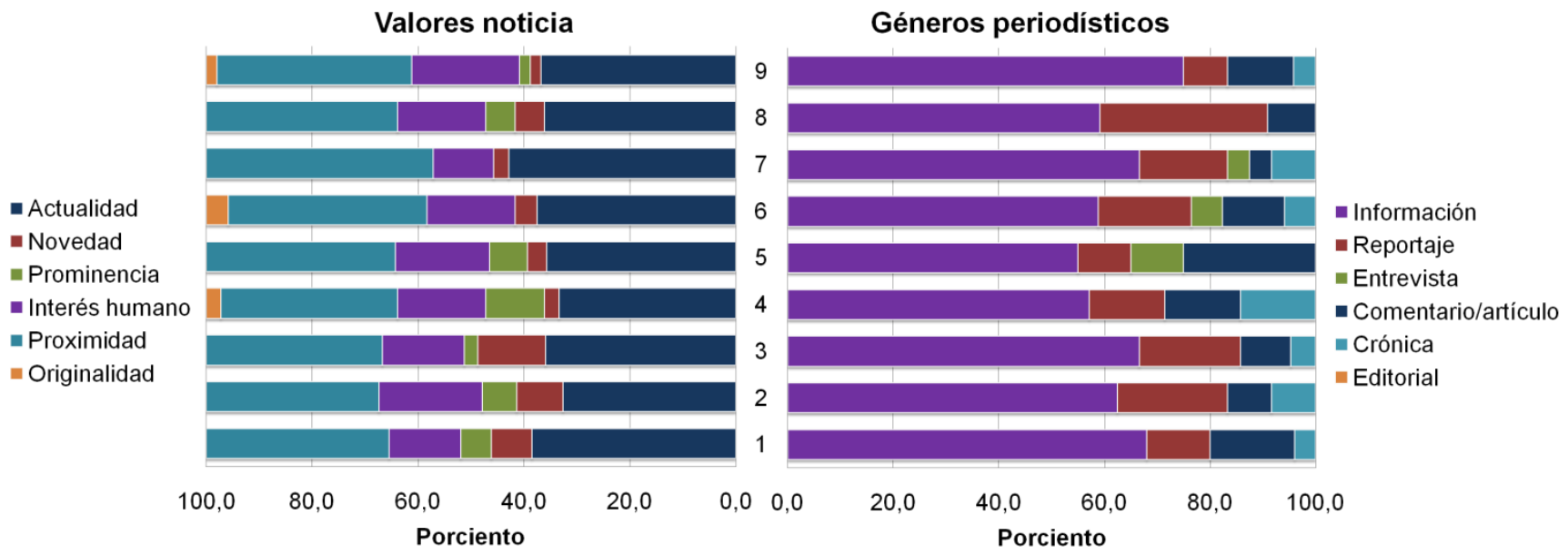

Fuente: elaboración propia

Un resultado similar se encontró en el telecentro, donde el género más utilizado es la Información con un 78,5\% de los trabajos publicados, y el Reportaje, con un 13,0\%. Los valores noticia más empleados fueron la proximidad $(39,0 \%)$ y la actualidad $(38,8 \%)$. Nótese cómo el uso de los géneros y los valores noticia en estos medios se diferencia un tanto, a pesar de que ambos poseen formatos similares. Al igual que ocurrió en el caso de la emisora, en el telecentro se mantiene estable en el tiempo la utilización de ambos elementos.

Figura 2 Uso de los géneros periodísticos y valores noticia en el telecentro Tele Turquino según las semanas de estudio

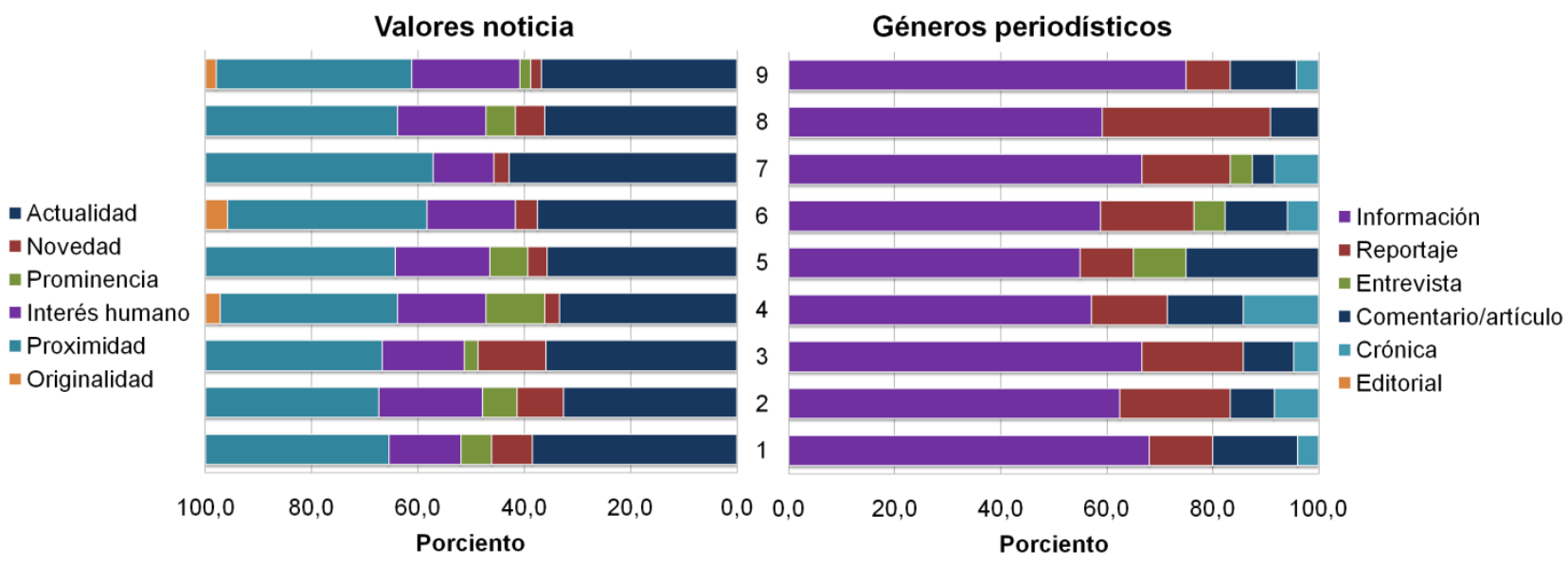

Fuente: elaboración propia

Por último, en el periódico los géneros más utilizados fueron la Información (63,6\%), el reportaje $(16,7 \%)$ y el comentario $(12,1 \%)$, lo que se corresponde con el empleo de valores noticia como la actualidad (36,5\%), el interés humano $(16,5 \%)$ y la proximidad $(35,6 \%)$. En este caso los géneros periodísticos responden principalmente al espacio disponible en el medio y a su función social: primeramente informar, y luego analizar los hechos noticiosos que se presentan, fundamentalmente dado su 
Muñiz Zúñiga, V.; Fonseca Valido, R. A. Uso de géneros periodísticos, valores noticia y fuentes de información en los medios de comunicación de Santiago de Cuba

carácter semanal.

Figura 3 Uso de los géneros periodísticos y valores noticia en el periódico Sierra Maestra según las semanas de estudio

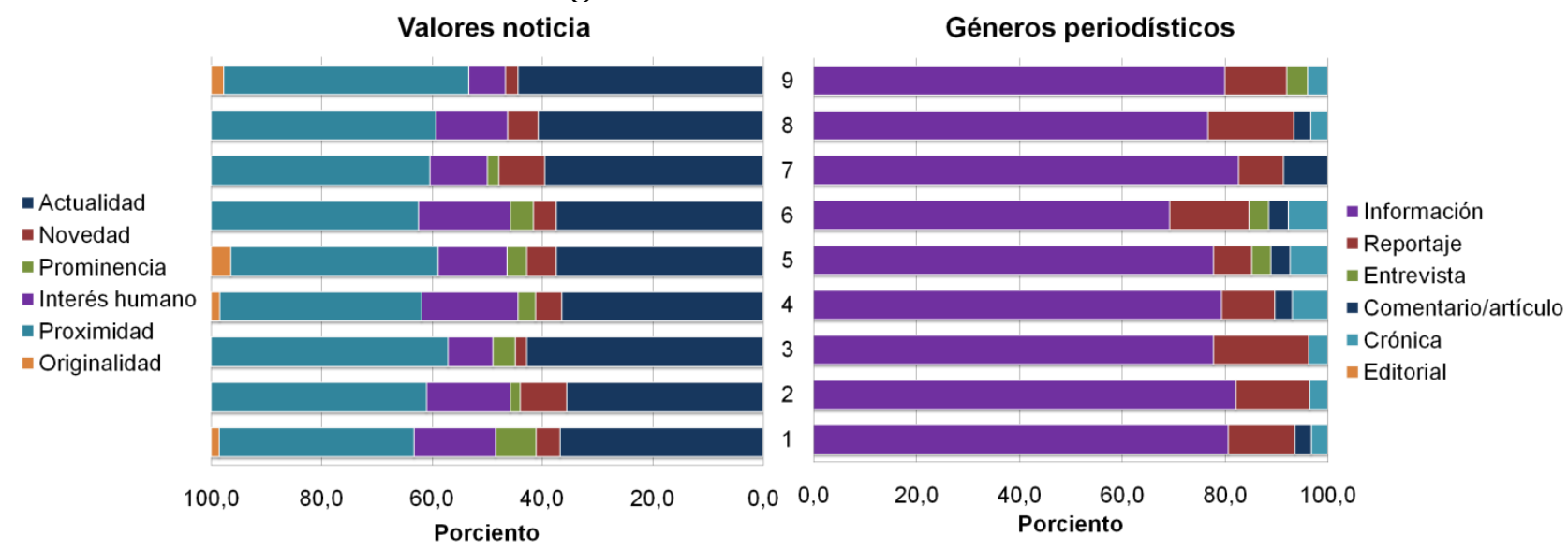

Fuente: elaboración propia

Las tablas 1 y 2 muestran los totales del uso de los géneros periodísticos y los valores noticia con sus respectivas frecuencias relativas y el resultado de la prueba binomial realizada con el objetivo de establecer el nivel de significación de cada uno durante las semanas del estudio. El nivel utilizado en ambos casos fue 0.05 .

Tabla 1 Porcentaje del uso de los géneros periodísticos y niveles de significación de la prueba binomial

\begin{tabular}{|c|c|c|c|c|c|c|c|c|c|}
\hline 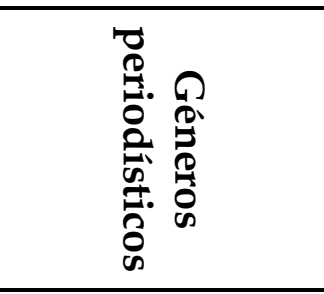 & 定 & $a^{e}$ & 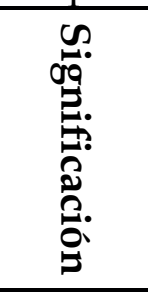 & $\begin{array}{l}0 \\
0 \\
0 \\
0 \\
2 \\
3 \\
0 \\
0 \\
0 \\
0 \\
0 \\
0\end{array}$ & $a^{e}$ & 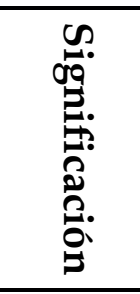 & 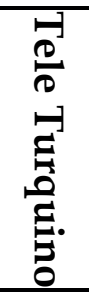 & $0^{\circ}$ & 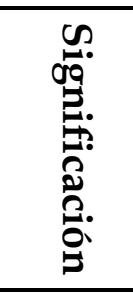 \\
\hline Información & $\begin{array}{c}25 \\
8\end{array}$ & 73,93 &, $000(a)$ & $\begin{array}{c}12 \\
6\end{array}$ & 63,64 &, $000(a)$ & 193 & 78,46 &, $000(a)$ \\
\hline Reportaje & 15 & 4,30 & $\begin{array}{c}\text {,326(b, } \\
\text { a) }\end{array}$ & 33 & 16,67 &, $000(a)$ & 32 & 13,01 &, $000(a)$ \\
\hline Entrevista & 28 & 8,02 & ,010(a) & 4 & 2,02 & $\begin{array}{l}, 028(b, \\
\text { a) }\end{array}$ & 3 & 1,22 & $\begin{array}{l}, 002(b, \\
\text { a) }\end{array}$ \\
\hline $\begin{array}{c}\text { Comentario/artí } \\
\text { culo }\end{array}$ & 39 & 11,17 &, $000(a)$ & 24 & 12,12 &, $000(a)$ & 7 & 2,85 & $\begin{array}{c}\text {,072(b, } \\
\text { a) }\end{array}$ \\
\hline Crónica & 8 & 2,29 & $\begin{array}{c}, 008(b, \\
\text { a) }\end{array}$ & 11 & 5,56 & ,404(a) & 11 & 4,47 & $\begin{array}{c}\text {,424(b) } \\
\text { a) }\end{array}$ \\
\hline Editorial & 1 & 0,29 & $\begin{array}{l}, 000(b, \\
\text { a) }\end{array}$ & 0 & 0,00 &, $000(a)$ & 0 & 0,00 &, $000(a)$ \\
\hline $\begin{array}{c}\text { Total } \\
\text { (a) } p>0.05 \\
(b, a) p<0.05\end{array}$ & $\begin{array}{c}34 \\
9\end{array}$ & $\begin{array}{c}100,0 \\
0\end{array}$ & & $\begin{array}{c}19 \\
8\end{array}$ & $\begin{array}{c}100,0 \\
0\end{array}$ & & 246 & $\begin{array}{c}100,0 \\
0\end{array}$ & \\
\hline
\end{tabular}




\section{Fuente: elaboración propia}

El análisis de contenido reveló que el porcentaje obtenido en el uso de los géneros y los valores noticia se mantienen de forma regular durante las 9 semanas estudiadas, lo que revela una posible permanencia temporal de estos elementos. Asimismo, el género más utilizado en estos órganos de prensa es la información, seguida de otros de opinión, como el comentario, y de interpretación, como el reportaje. Este comportamiento se debe fundamentalmente a rutinas de uso en los medios, estrechamente ligadas a la práctica profesional de los periodistas. Por ejemplo, en la radio señalaron en las entrevistas que "la vorágine de trabajo provoca que hagamos lo mismo y lo mismo, el casete se va repitiendo todos los meses." En tanto los de la televisión opinan que le falta profundidad al periodismo que realizan, y que se deben tratar los temas de diferentes maneras y no como siempre se ha realizado, pues de cierta forma los receptores están cansados de ver lo mismo.

Por ello, los entrevistados explican que a pesar de que las fuentes de información provean de temas importantes a los medios de comunicación, también hay que inyectarle nuevas propuestas a las agendas para que estas no se comporten de manera estática -o semi estática-, como ha estado ocurriendo en varios casos.

Tabla 2 Porcentaje del uso de los valores noticia y niveles de significación de la prueba binomial

\begin{tabular}{|c|c|c|c|c|c|c|c|c|c|}
\hline 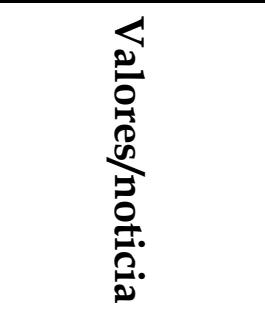 & 3 & $a^{0}$ & 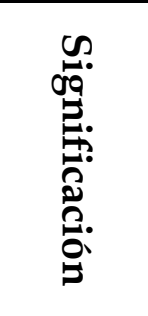 & 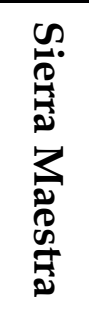 & $a^{0}$ & 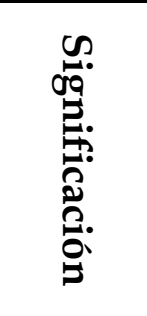 & 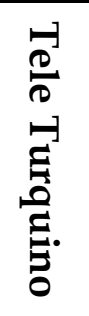 & $a^{0}$ & 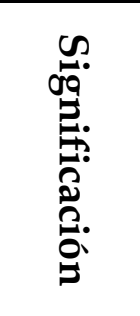 \\
\hline Actualidad & $\begin{array}{c}33 \\
2\end{array}$ & $\begin{array}{c}39,0 \\
1\end{array}$ &, $000(\mathrm{a})$ & 126 & $\begin{array}{c}36,5 \\
2\end{array}$ &, $000(a)$ & 190 & $\begin{array}{c}38,7 \\
8\end{array}$ &, $000(a)$ \\
\hline Novedad & 47 & 5,52 & ,262(a) & 20 & 5,80 & ,280(a) & 25 & 5,10 & ,488(a) \\
\hline Prominencia & 42 & 4,94 & $\begin{array}{c}506(\mathrm{~b}, \\
\text { a) }\end{array}$ & 16 & 4,64 & $\begin{array}{c}441(\mathrm{~b}, \\
\text { a) }\end{array}$ & 15 & 3,06 & $\begin{array}{c}, 025(b, \\
\text { a) }\end{array}$ \\
\hline $\begin{array}{l}\text { Interés } \\
\text { humano }\end{array}$ & 99 & $\begin{array}{c}11,6 \\
3\end{array}$ &, $000(\mathrm{a})$ & 57 & $\begin{array}{c}16,5 \\
2\end{array}$ &, $000(a)$ & 64 & $\begin{array}{c}13,0 \\
6\end{array}$ &, $000(a)$ \\
\hline Proximidad & $\begin{array}{c}32 \\
2\end{array}$ & $\begin{array}{c}37,8 \\
4\end{array}$ &, $000(\mathrm{a})$ & 123 & $\begin{array}{c}35,6 \\
5\end{array}$ &, $000(a)$ & 191 & $\begin{array}{c}38,9 \\
8\end{array}$ &, $000(a)$ \\
\hline $\begin{array}{c}\text { Originalida } \\
\mathrm{d}\end{array}$ & 9 & 1,06 & $\begin{array}{l}, 000(b, \\
\text { a) }\end{array}$ & 3 & 0,87 & $\begin{array}{l}, 000(b, \\
\text { a) }\end{array}$ & 5 & 1,02 & $\begin{array}{l}, 000(b, \\
\text { a) }\end{array}$ \\
\hline $\begin{array}{c}\text { Total } \\
\begin{array}{c}(a) p>0.05 \\
(b, a) p<0.05\end{array}\end{array}$ & $\begin{array}{c}85 \\
1\end{array}$ & $\begin{array}{c}100 \\
00\end{array}$ & & 345 & $\begin{array}{c}100 \\
00\end{array}$ & & 490 & $\begin{array}{c}100 \\
00\end{array}$ & \\
\hline
\end{tabular}

Fuente: elaboración propia 
Los valores noticia más empleados en los tres medios fueron la actualidad-lo que se relaciona con el uso de la información como género-, la proximidad, debido fundamentalmente a la naturaleza de los productos analizados, que se encuentran dirigidos a los habitantes de la provincia, porque precisamente son medios con alcance provincial. Esto determina que la mayor parte de los productos periodísticos que se publican tengan un contenido cercano a la experiencia y a las condiciones de vida de las personas, y no solo geográficamente, como se puede inferir. Este elemento también está ligado al uso del interés humano como valor noticia, pues en aras de hacer un producto más atractivo los periodistas recurren con frecuencia a historias de vida de personas que pueden suscitar el consumo de los trabajos publicados.

Para contrastar la información obtenida con el análisis de contenido, se aplicó una encuesta en la cual se les interrogó a los periodistas de los medios analizados cuál era el uso que ellos daban a los géneros periodísticos y los valores noticia. En cuanto a la opinión sobre la posibilidad de ejercer la crítica en el medio en el cual trabajaban, la mayor parte apuntó que era regular $(35,29 \%$ en el periódico, $50,00 \%$ en la radio y $55,56 \%$ en la televisión), aunque en el periódico la mayoría $(47,06 \%)$ consideró que era buena. En los tres medios los periodistas consideraron que era mala en menor medida. Este resultado indica que realmente no existen trabas institucionales sustanciales que impidan realizar trabajos con un enfoque crítico, pues como señalaron en las entrevistas "los directivos, las fuentes y el Partido Comunista de Cuba potencian el empleo de géneros y trabajos periodísticos más críticos, aunque esto no sucede con todas las fuentes". Evidentemente existen fuentes de información que son más cerradas a la hora de brindar información, y limitan en ocasiones a que el periodista fustigue elementos negativos en su quehacer. Ello condiciona el uso de géneros periodísticos interpretativos y de opinión.

Un elemento observable en las entrevistas fue el espacio disponible para la publicación: "muchas veces los periodistas queremos hacer un trabajo más profundo, extenso, pero por un problema de espacio no lo publican". Esto sucede mayormente en el periódico en su versión impresa, pues solo cuentan con 8 páginas en formato tabloide una vez a la semana. Sin embargo, en la radio influyen las rutinas de edición de los trabajos, la planificación de los estudios de grabación muchas veces atenta contra la producción de estos géneros más "delicados" por la realización sonora que llevan. En la televisión "los periodistas suelen planificarse, pero igualmente el horario de los cubículos de edición es escaso, pues a veces el periodista solo cuenta con una hora para editar un trabajo de tres minutos, y sabemos que en dependencia del tema y los recursos visuales se necesita más tiempo."

Por otra parte, a los periodistas se les interrogó en la encuesta sobre la frecuencia del uso de los géneros periodísticos y los valores noticia en su medio según su opinión. La figura 4 muestra el criterio de los profesionales del periódico; obsérvese que los resultados cuantitativos coinciden parcialmente con aquellos que obtuvimos en el análisis de contenido. Lógicamente, este resultado está relacionado además con la concepción que cada periodista tenga de los valores noticia y de cuál debe ser su empleo en los trabajos que elaboran, recordemos que este es un producto socialmente creado. 
Muñiz Zúñiga, V.; Fonseca Valido, R. A. Uso de géneros periodísticos, valores noticia y fuentes de información en los medios de comunicación de Santiago de Cuba

Figura 4 Frecuencia del uso de los géneros periodísticos y los valores noticia según los encuestados del periódico

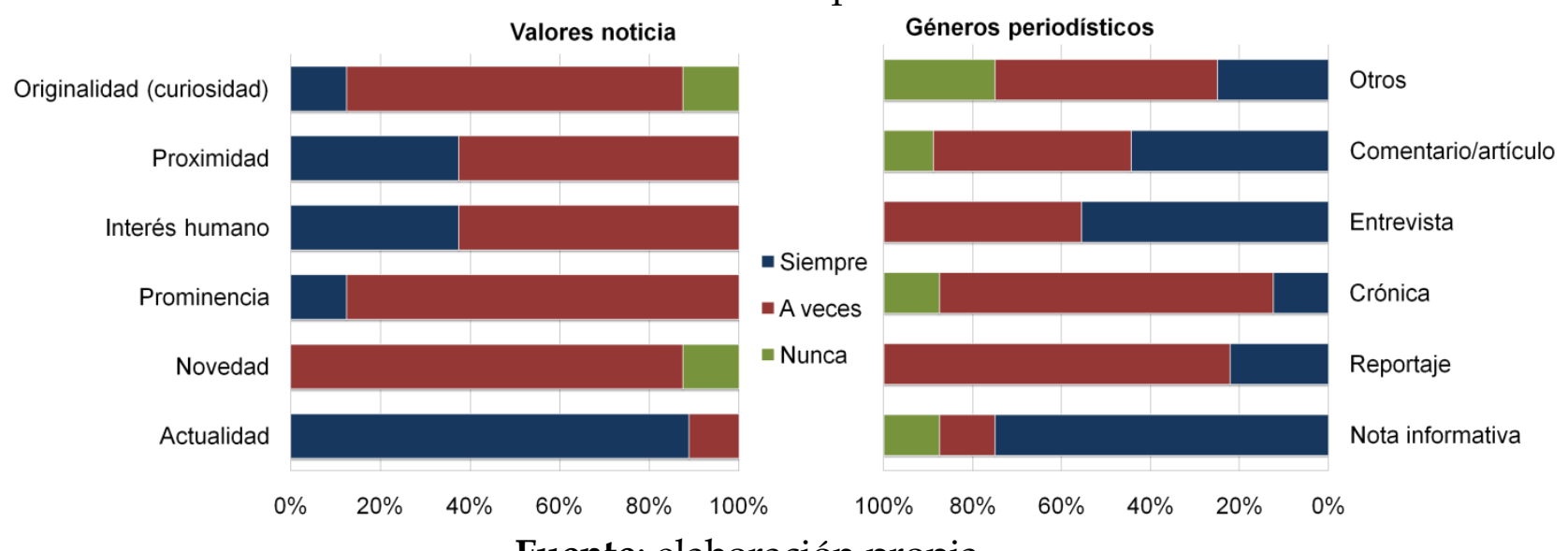

Fuente: elaboración propia

Sin embargo, lo que encontramos en el caso del periódico en cuanto a la percepción fue muy similar a lo que hallamos en la emisora CMKC. Nótese en la figura 5 que los periodistas consideran que siempre utilizan la inmediatez y la proximidad como valores noticia, lo que se encuentra en consonancia con el tipo de medio que evaluamos.

Figura 5 Frecuencia del uso de los géneros periodísticos y los valores noticia según los encuestados de la emisora CMKC

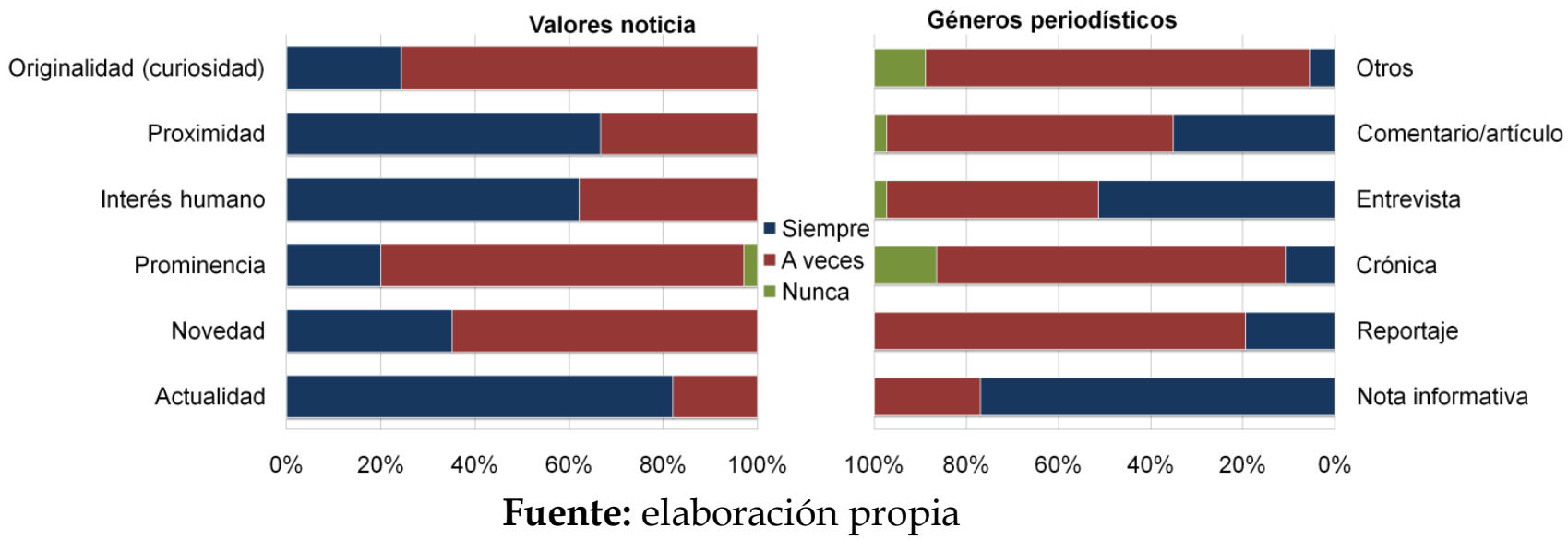

Esta regularidad en el resultado responde a una concepción homogénea y regular del fenómeno, lo que indica que en los medios de comunicación existen condiciones similares de producción, independientemente del formato en el que se elaboren los trabajos. La figura 6 permite observar esta particularidad: 
Figura 6 Frecuencia del uso de los géneros periodísticos y los valores noticia según los encuestados de la televisión

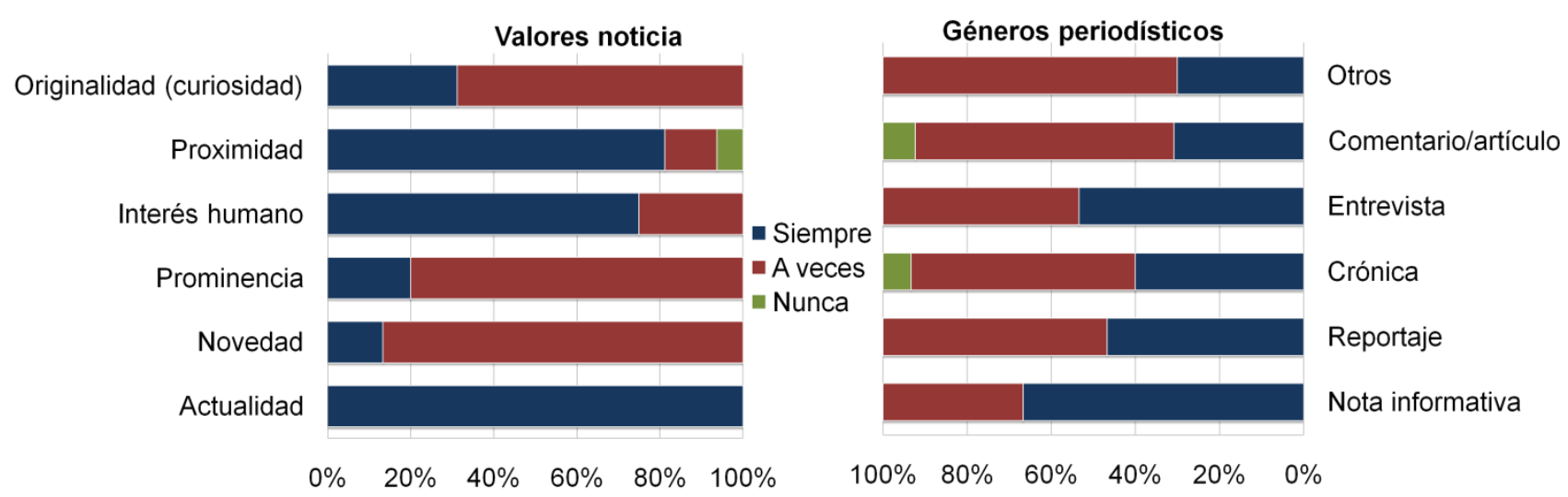

Fuente: elaboración propia

Hasta el momento, tanto el análisis de contenido como la encuesta y las entrevistas realizadas revelan una rutina del uso de los géneros periodísticos y los valores noticia con características muy similares en los órganos de prensa estudiados, lo que conlleva a la conclusión de que en la provincia no solo existe una concepción unificada acerca del uso de ambos elementos, sino de su importancia para enriquecer la labor en los medios. Evidentemente, su empleo está relacionado con factores que emanan de la construcción de la noticia en cada órgano, como la propia organización del trabajo, el espacio y la disponibilidad de recursos materiales, las fuentes tratadas por los periodistas y la capacidad que estos tengan para hacer un producto con mayor calidad.

\subsection{Uso de las fuentes de información}

Las fuentes de información en el trabajo periodístico constituyen una poderosa herramienta que permite la mayor cantidad de integración de elementos en un trabajo noticioso. En el presente estudio mostramos los resultados de la triangulación metodológica realizada a partir del análisis de contenido, la encuesta aplicada a los periodistas y la entrevista en la cual se les interrogó sobre la relación con las fuentes institucionales, los motivos por los cuales consultaban una fuente y no otra, etc.

En las entrevistas los periodistas señalaron que las fuentes de información resultan importantes para el trabajo que realizan porque ofrecen disímiles visiones, perspectivas, contenidos informativos, experiencias que enriquecen su labor; asimismo, sirven para verificar, preparar, completar y orientar el proceso de construcción de la noticia, porque forman parte, de uno u otro modo del acontecimiento noticioso.

El 76,47\% de los sujetos encuestados en el periódico señaló que siempre utiliza como fuente a las instituciones estatales, seguida de un 47,06\% que afirma emplear el criterio del pueblo. Por ello, en el análisis de contenido el 48,7\% de las fuentes empleadas eran precisamente las institucionales, seguidas de las personales (el 
público), con un 15,8\%. La figura 7 muestra los resultados obtenidos en el análisis de contenido a los medios, y la figura 8 muestra las respuestas de los periodistas en la encuesta:

Figura 7 Uso de las fuentes de información por parte de los periodistas según el análisis de contenido

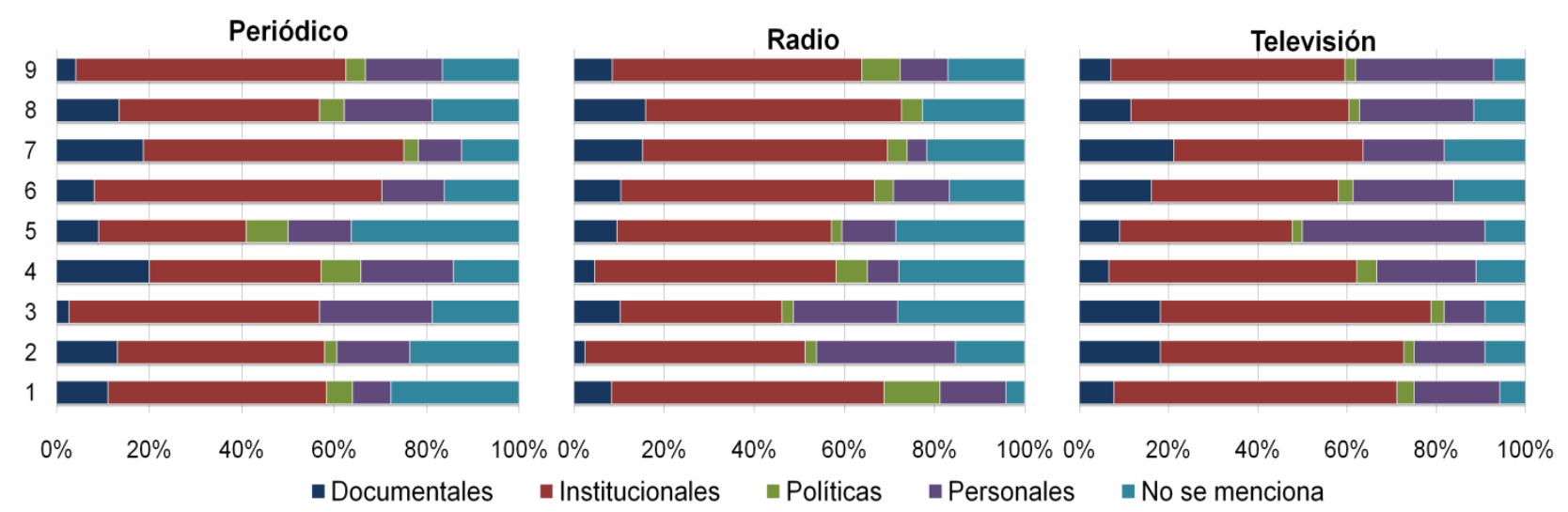

Fuente: elaboración propia

En la radio sucede a la inversa, pues el $55,56 \%$ de los periodistas afirma utilizar como fuente al pueblo santiaguero, y el 50,00\% a las instituciones estatales. Sin embargo, en el análisis de contenido el $52,5 \%$ de las fuentes utilizadas eran estatales, y solo un $12,4 \%$ personales.

Figura 8 Uso de las fuentes de información por parte de los periodistas según las encuestas realizadas

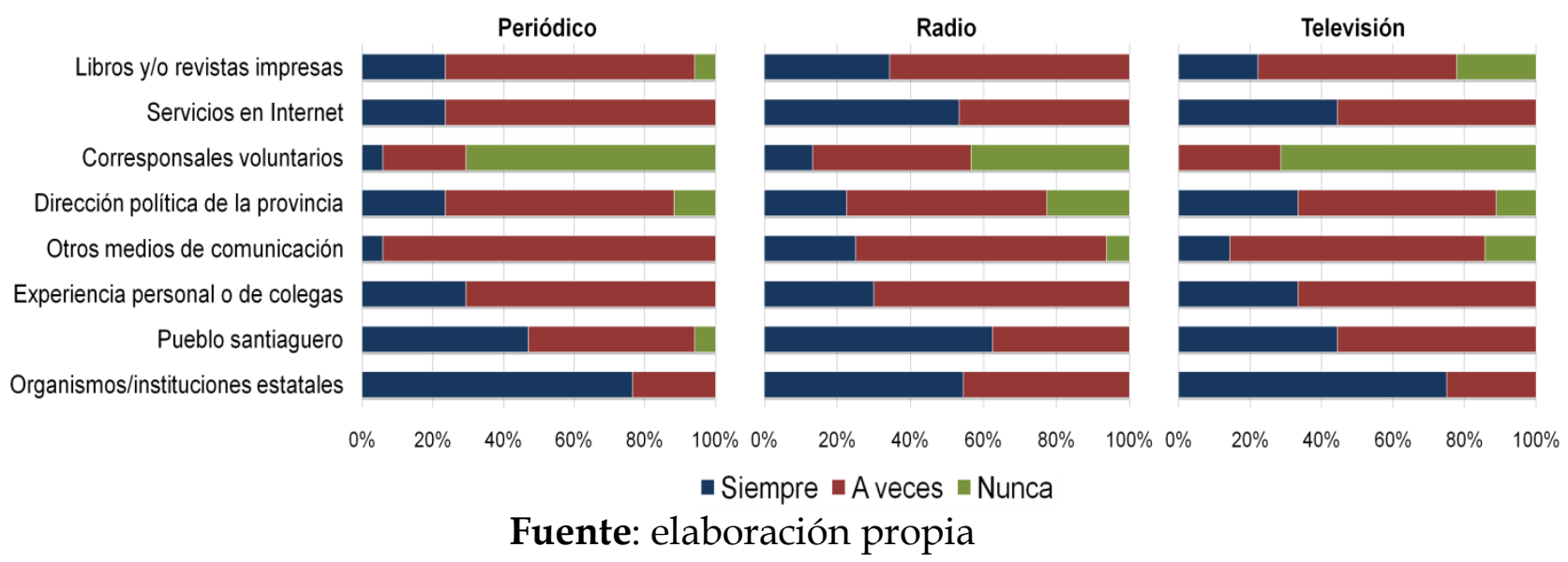

Por último, en el caso del telecentro el $66,67 \%$ de los periodistas señaló que siempre utiliza como fuentes los organismos del Estado, y que a veces recurre a la población o Internet (55,56\% respectivamente).

En la conformación de la agenda de un periodista o un medio inciden muchas fuentes informativas, cuyo empleo puede variar en dependencia del interés personal del periodista, o del medio en sentido general. Por ejemplo, el 23,53\% de los encuestados en el periódico afirma que siempre utiliza como fuentes a la dirección política de la provincia, los sitios web de Internet y libros y/o revistas impresas; en tanto, en la radio, el $25,00 \%$ dice basarse en la experiencia personal o de colegas, y el $22,22 \%$ afirma utilizar lo que publican otros medios de comunicación. Esto se debe a 
que las fuentes documentales son consideradas como "imprescindibles" en el trabajo de los periodistas, principalmente por la necesidad de hacer la labor periodística con la inmediatez que requiere; por la no disponibilidad o accesibilidad a ciertos documentos y las especificidades del medio de trabajo (televisión, prensa escrita o digital, radio).

Ahora bien, en las entrevistas realizadas se pudo determinar que el uso de las fuentes de información institucionales se debe principalmente a la búsqueda de confiabilidad de los datos, la especialización de la fuente- condicionada por el elemento anterior- y el nivel jerárquico que esta ocupa. En este aspecto, cabe destacar que algunos valoran el uso de una fuente determinada atendiendo a los hechos abordados: "quizás una persona de la calle, fuera de una institución, pueda hablar mejor de la calidad del pan que el propio administrador de la panadería". Por ello, destacamos que el uso de las fuentes debe ir aparejado a la intención del periodista.

Por otra parte, observemos que los datos del análisis de contenido no coinciden con los criterios brindados en las encuestas, lo que nos lleva a considerar que los periodistas mantienen una percepción irreal de su relación con el público, aunque sí son conscientes de que emplean más fuentes institucionales que de otra índole.

Por último, en el análisis de contenido existe un porciento considerable $(19,9 \%$ en la radio, $20,1 \%$ en el periódico, y $10,4 \%$ en la televisión) de fuentes que no se mencionan, o sea, que no aparecen citadas en los trabajos periodísticos; además, de que el porcentaje de empleo de otras fuentes aquí mencionadas es significativamente menor al de las instituciones, lo que presumiblemente indica que es pobre el uso de varias fuentes de información en el mismo producto comunicativo.

En cuanto al acceso a las fuentes de información institucionales, el RSB 232, que regula entre otros elementos la relación entre los periodistas y las fuentes de información, referencia en su texto la principal dificultad que afrontan nuestros comunicadores en cuanto al trabajo con estas. Dirigentes y funcionarios estatales a todos los niveles suelen evadir el contacto con la prensa, porque no se consideran autorizados para difundir determinadas informaciones de interés que, realmente, no constituyen secretos, y en ocasiones, al acceder a ello, imponen excesivas limitaciones. De hecho, el documento citado fue escrito en el 2007, y aun en la actualidad no se han resuelto estas dificultades.

Los profesionales opinan que el trabajo con las fuentes es de gran ayuda, porque entre ellos y las instituciones se crea un vínculo de trabajo estrecho. Esto es válido en cierta medida, pues actualmente existen muchos periodistas cuyas fuentes mantienen un contacto directo, una comunicación estrecha; y entonces la información fluye mejor.

Pero desgraciadamente no todas las fuentes hacen lo que debieran, incluso a veces se convierten en el principal obstáculo, no dando lo necesario o tergiversando los datos. Sucede también que determinados organismos que están implicados en algunos materiales se atribuyen la revisión de estos una vez terminados; acción permisible si existe algún término especializado, y siempre que no se le diga al periodista qué escribir o no. Sin embargo, en situaciones como esta, es de ayuda el papel orientador del Partido Comunista de Cuba, mediando muchas veces para que la información sea finalmente suministrada: "al poner una traba al periodista, también se la ponen al Partido. Por lo general las instituciones colaboran." 
Por último, los periodistas se refirieron en las entrevistas a la sectorialización como un elemento fundamental en la relación con las fuentes. En este sentido los criterios no coincidieron entre sí, pues mientras algunos opinan que el acomodamiento en una determinada fuente es perjudicial para el trabajo periodístico- debido a las relaciones interpersonales y la confianza que se va ganando con el tiempo-; otros ven este aspecto como algo positivo, pues para ellos la comunicación abierta con la fuente es la mejor opción.

\section{CONCLUSIONES}

Luego del análisis realizado en el presente estudio podemos arribar a las siguientes conclusiones: el empleo de los géneros periodísticos informativos, de opinión e interpretativos en los trabajos publicados por los medios de comunicación de la provincia está determinado por rutinas establecidas al interior de cada órgano, pero que funcionan de manera similar en todos. Por ello en el periódico Sierra Maestra, la emisora CMKC y el telecentro Tele Turquino podemos observar que los géneros más frecuentes son la información, el reportaje y el comentario.

Esto se encuentra relacionado con el uso de los valores noticia, como herramienta para dotar al trabajo periodístico de elementos atractivos para el público y otros medios. En ese sentido, los valores más explotados son la inmediatez, la proximidad y el interés humano, lo que pudo observarse en los resultados del análisis de contenido realizado a los medios. En la triangulación de estos datos con las encuestas y la entrevista a los periodistas pudimos apreciar que los profesionales conciben de manera similar igualmente la utilidad de estos elementos, sobre todo de los valores noticia. Por ello, encontramos criterios parecidos en los resultados de estas técnicas.

Se evaluó que la relación de los periodistas santiagueros con las fuentes de información está sometida a elementos como el tipo de fuente que utilizada en los trabajos periodísticos, y las características o particularidades estructurales y organizativas de los medios. En sentido general predomina el uso de las fuentes documentales en búsqueda del equilibrio y la obtención de un producto informativo más acabado. Se pudo comprobar que aunque el intercambio con las fuentes orales o vivas (sean o no especializadas en un determinado tema de esfera o sector) es manejable, no descarta el hecho de que tenga cierto grado de incertidumbre y de indisponibilidad de algunas. Se concibe asimismo que la labor en los medios de comunicación fuese imposible sin la contribución de las fuentes, pues sin estas la información es pobre, carente de veracidad, fiabilidad y nivel de comprobación.

\section{REFERENCIAS}

Aguirre, C., \& Bernal, J. D. (2015). Contenidos periodísticos digitales: hacia un modelo de medición de calidad. Revista ComHumanitas, 5(1), 75-90.

Alcaraz, L. G. (2012). El cambio climático en la prensa local. Agenda informativa, valores noticiosos y encuadres periodísticos en dos diarios argentinos. Razón y palabra, 80, 36-29. 
Muñiz Zúñiga, V.; Fonseca Valido, R. A. Uso de géneros periodísticos, valores noticia y fuentes de información en los medios de comunicación de Santiago de Cuba

Alsina, M. R. (1993). La construcción de la noticia. Barcelona: Paidós.

Andrade, E. S. (2015). La realidad por escrito. Reflexiones en torno al análisis del discurso periodístico. Comunicación y Sociedad, 40.

Asociación de la Prensa de Madrid. (2013). Informe anual de la profesión periodística. Recuperado de http://www.apmadrid.es/publicaciones/informe-anual-de-laprofesion-periodistica.

Brown, J. D., Bybee, C. R., Wearden, S. T., \& Straughan, D. M. (1987). Invisible power: Newspaper news sources and the limits of diversity. Journalism and Mass Communication Quarterly, 64(1), 45.

Builes, I. A. (1996). La influencia de las rutinas productivas en la construcción del acontecer nacional: el caso de Siglo 21, periódico de Guadalajara, México. Comunicación y Sociedad, 28, 83-114.

Carballosa, B. R., Pavón, L. B., \& Rodríguez, E. V. (2016). La calidad de los productos periodísticos: una experiencia para su evaluación. Razón y palabra, (92), 13-17.

Cardoso, H. (2008). Investigación y creación periodística. La Habana: Editorial Pablo de la Torriente.

Cedillo, G. R., \& Carretero, A. B. (2015). Periodismo lento (slow journalism) en la era de la inmediatez: Experiencias en Iberoamérica. El profesional de la información, $24(4), 451-462$.

Colunga, M. (2011). Mediaciones sobre el contenido de los medios que inciden en la relación entre la agenda mediática del periódico Adelante y la agenda pública de sus lectores potenciales. (Tesis inédita de licenciatura). Universidad de Camagüey, Cuba.

Comité Central del Partido Comunista de Cuba. (2007). Orientaciones del Buró Politico del Comité Central del Partido Comunista de Cuba para incrementar la eficacia informativa de los medios de comunicación masiva del país. (Documento limitado).

De la Piscina, T. R., Gorosarri, M. G., Zabalondo, B., \& Agirre, A. (2014). Periodismo de calidad en tiempos de crisis: Un análisis de la evolución de la prensa europea de referencia (2001-2012. [Quality journalism in times of crisis: An analysis of the evolution of the European reference press (2001-2012)]. Revista Latina de Comunicación Social, 69, 248.

De la Torre, L., \& Téramo, M. T. (2015). La calidad de la información periodística: estrategias para su observación. Coincidencias y divergencias entre los medios y el público. Estudios sobre el Mensaje Periodístico, 21, 135-144. 
Díaz, N. S., González, M. D. M., Gutiérrez-Coba, L., \& Cardona, A. S. (2016). Análisis de la cobertura de noticias sobre alcoholismo y drogadicción en la prensa colombiana. Health and Addictions/Salud y Drogas, 16(1), 41-48.

Falla, J. M. P. P., \& Ramírez, R. M. R. (2015). Tratamiento Periodístico de las Noticias en Diarios. Casos de Niños y Adolescentes en Conflictos con la Ley. Lambayeque-2013. Revista Científica PAIAN, 6(1).

García, J (2013). Revolución, socialismo, periodismo. La prensa y los periodistas cubanos ante el siglo XXI. La Habana: Pablo de la Torriente.

Golding, P., \& Elliott, P. R. C. (1979). Making the news. Longman Publishing Group.

Gómez, L. P. (2016). El papel del periodista en la información política electoral y uso de Twitter en la difusión de contenidos. Revista de la Asociación Española de Investigación de la Comunicación, 3(5).

Gomis, L. (1991). Teoría del periodismo. Cómo se forma el presente. España: Paidós Comunicación.

González, A. D. L., Robles, F. A., Prieto, M. C., \& Avilés, J. A. G. (2015). Ranking de innovación periodística 2014 en España: Selección y análisis de 25 iniciativas. El profesional de la información, 24(3), 235-245.

González, J. M. (2016). Periodismo Interpretativo: precisiones en torno a un género. Cuadernos info, 3.

Hernández, G. M. (2015). Los géneros periodísticos de opinión y los valores. Estudios sobre el Mensaje Periodístico, 21(2), 1099-1110.

Ibáñez, D. B., Alonso, M. O., \& Hernández, S. (2015). Análisis de contenido informatizado para las aproximaciones al concepto de objetividad de los profesionales de la información de la cultura periodística de España. Revista ABRA, 35(50), 1-16.

Lippmann, W. (1969). The nature of news. Mass Media and Communication. Nueva York: Hastings House.

López Rabadán, P., \& Casero Ripollés, A. (2014). La información periodística de portada en España: evolución histórica de fuentes, agendas y encuadres (19802010)/The cover news reporting in Spain: historical evolution of sources, agenda and frames (1980-2010). Historia y Comunicación Social, 19, 457. 
Muñiz Zúñiga, V.; Fonseca Valido, R. A. Uso de géneros periodísticos, valores noticia y fuentes de información en los medios de comunicación de Santiago de Cuba

Macías, R. A. G. (2015). Del amor al odio: rutinas y lenguaje usado en la cobertura de la Selección Mexicana en Brasil 2014. Global Media Journal México, 12(23).

Martini, S. (2000). Periodismo, noticia y noticiabilidad (Vol. 4). Editorial Norma.

McCombs, M. (2006). Estableciendo la agenda: el impacto de los medios en la opinión pública y en el conocimiento. España: Paidós.

Mompart, J. L. G., \& Sampio, D. P. (2013). Métodos y técnicas de análisis y registro para investigar la calidad periodística. In Investigar la Comunicación hoy. Revisión de politicas científicas y aportaciones metodológicas: Simposio Internacional sobre Política Científica en Comunicación (pp. 771-787). Facultad de Ciencias Sociales, Juridicas y de la Comunicación.

Mompart, J. L. G., Lozano, J. F. G., \& Sampio, D. P. (2015). La calidad periodística en España según la percepción de los periodistas. Estudios sobre el Mensaje Periodístico, 21, 13-30.

Mompart, J. L. G., Lozano, J. F. G., \& Sampio, D. P. (Eds.). (2013). La calidad periodística: teorías, investigaciones y sugerencias profesionales (Vol. 26). Universitat de València.

Orosa, B. G., \& Santorum, S. G. (2012). La conformación de valores noticia en un contexto de conflicto. Análisis pragmático de su funcionamiento en la información sobre el narcotráfico en México y el feminicidio de Ciudad Juárez. Estudios sobre el mensaje periodístico, 18(2), 491-511.

Pineda, A., \& Molero, L. (2015). Genes, visibilidad mediática y discursos emergentes. Análisis semántico-pragmático de notas cortas. Enl@ ce, 12(1).

Reyes, A. R., González, A. E., Torrijos, J. L. R., \& Gordillo, M. D. M. G. (2015). La calidad de los medios y el uso de fuentes periodísticas en la prensa local de referencia en España. Estudios sobre el Mensaje Periodístico, 21, 85-100.

Reyes, L. (2006). Manual de fuentes de información. La Habana: Editorial Pablo de la Torriente.

Ribeiro, J. C. (2016). De la columna a la información corta móvil: análisis de los géneros periodísticos en los j-blogs políticos de Brasil y España. Chasqui. Revista Latinoamericana de Comunicación, 129, 265-283.

Rosquete D. (2010). Estudio del proceso de establecimiento de la agenda mediática sobre el acontecer nacional del SITVC. (Tesis de licenciatura). Universidad de La Habana, Cuba. 
Serrano, M., \& Piñuel, J. L. (1982). Teoría de la comunicación: epistemología y análisis de la referencia. Cuadernos de la comunicación, Madrid-España.

Sigal, L. V. (1973). Reporters and officials: The organization and politics of news reporting. Lexington: Mass Heath.

Teramo, M. T. (2006). Calidad de la información periodística en Argentina. Estudio de diarios y noticieros. Palabra Clave, 9(1), 57-84.

Toledo, H. C., Medina, H. S., \& Hernández, M. R. (2015). Mediación de las lógicas de producción hipermedia en el uso de la web 2.0 en Radio Sancti Spíritus. Un estudio de caso cubano. Mediaciones Sociales, (13), 3-36.

Vicente, B. R., Rojas, M. M. H., \& Vicente, E. J. R. (2016). Convergencias narrativas de la radio en Cuba. Razón y palabra, (92), 55-22.

\section{AUTORES}

\section{Viviana Muñiz Zúñiga}

Licenciada en Periodismo, graduada en la Universidad de Oriente en Cuba. Investiga desde el 2013 los procesos de construcción y establecimiento de la agenda mediática en Cuba; la implementación de las Política Informativa y Editorial y la construcción de la noticia en los medios cubanos.

http:/ / www.redalyc.org/autor.oa?id=14461

\section{Rafael Ángel Fonseca Valido:}

Doctor en Ciencias de la Comunicación. Es graduado de la universidad de Oriente en Cuba. Investiga los procesos de producción-emisión y recepción-consumo de mensajes mediáticos, especialmente de carácter periodístico; así como la optimización de los órganos de prensa cubanos.

https://www.researchgate.net/scientific-

contributions/2088522548_Rafael_Angel_Fonseca_Valido

